Forschende
Komplementärmedizin
Research in
Complementary Medicine

Complementary Medicine
D. Melchart ${ }^{\mathrm{a}}$

S. Hager $^{\mathrm{b}}$

J. Z. Liao $^{\mathrm{b}}$

K. Linde

W. Weidenhammer ${ }^{\mathrm{a}}$

a «Münchener Modell», Zentrum für naturheilkundliche Forschung, Technische Universität/Ludwig-Maximilians-Universität, München

b TCM-Klinik, Kötzting

\section{Beobachtungsstudien im Rahmen eines naturheilkundlichen Klinikverbunds}

\author{
Teil II: Detaillierte Ergebnisse \\ der Klinik für Traditionelle Chinesische Medizin Kötzting
}

\section{Schlüsselwörter}

Beobachtungsstudie · Qualitätssicherung - Traditionelle chinesische Medizin · Akupunktur - Chinesische Arzneimitteltherapie · Schmerz

\section{Zusammenfassung}

Ziele: Beschreibung von Patientenmerkmalen, diagnostischen und therapeutischen Leistungen und Ergebnisdaten einer Klinik für traditionelle chinesische Medizin. Design: Prospektive Beobachtungsstudie mit 12 Monaten Follow-up. Patienten: Alle 667 während des Dokumentationszeitraums stationär aufgenommenen $\mathrm{Pa}-$ tienten (Follow-up-Quote nach 12 Monaten 65,8\%). Zielkriterien: Soziodemographische Patientenmerkmale, Diagnosen, Krankheitsdauer, Art und Häufigkeit diagnostischer und therapeutischer Massnahmen, Beschwerdeintensität, Therapieerfolgsbeurteilung, Lebensqualität. Ergebnisse: Etwa zwei Drittel der Patienten litten unter chronischen Schmerzsyndromen (häufigste Einzeldiagnosen waren Migräne, Lumbago und Nacken- oder Kopfschmerzen). $72,3 \%$ der Patienten waren Frauen; die mediane Dauer der Hauptbeschwerden betrug 7 Jahre. Fast alle Patienten erhielten Akupunktur und chinesische Arzneimitteltherapie. 50,3\% der Patienten beurteilten den Therapieerfolg bei Entlassung als gut oder sehr gut, nach 12 Monaten waren es 55,6\%. Sowohl die Intensität der Hauptbeschwerden wie auch die Lebensqualität verbesserten sich deutlich nach Therapie. Nach 12 Monaten waren die Werte etwas rückläufig, lagen jedoch noch immer deutlich unter den Ausgangswerten. Schlussfolgerungen: Die Patienten der Klinik profitieren von der stationären Behandlung. Ohne entsprechende und diagnosespezifische Daten aus anderen Einrichtungen kann jedoch nicht beurteilt werden, wie die Ergebnisse im Vergleich einzuschätzen sind.

\section{Key Words}

Observational study $\cdot$ Quality management · Traditional Chinese medicine $\cdot$ Acupuncture $\cdot$ Chinese herbal therapy $\cdot$ Pain

\section{Summary}

Observational Studies in a Network of Hospitals Using Complementary Medicine. Part II: Detailed Results from the Hospital for Traditional Chinese Medicine in Kötzting

Objective: To collect information on patients, interventions and outcomes in a hospital for traditional Chinese medicine in Germany. Design: Prospective observational study with 12 months followup. Patients: All 667 consecutive patients admitted for in-patient treatment in the hospital between December 1994 and July 1995 were documented. The follow-up rate after 12 months was $65.8 \%$. Outcome Measures: Sociodemographic data, diagnoses, duration of complaints, type and frequency of diagnostic and therapeutic interventions, intensity of complaints, assessment of the therapeutic success, and quality of life. Results: About two thirds of the patients suffered from chronic pain syndromes (the most frequent single diagnoses were migraine, lumbago, and neck pain/headaches). $72.3 \%$ of the patients were female; the median duration since the onset of the disease was 7 years. Almost all patients received acupuncture and treatment with traditional Chinese drugs. $50.3 \%$ assessed the therapeutic success as good or very good at discharge; after 12 months this rate was $55.6 \%$. Both intensity of main complaints and psychic and physical aspects of quality of life improved after treatment. After 12 months the improvement was less distinct but still significant. Conclusions: The in-patient treatment provided a clear benefit to the patients. Without a valid comparison with an alternative treatment little can be concluded about comparative effectiveness and efficiency of a treatment in the hospital for traditional Chinese medicine. 


\section{Einleitung}

Die traditionelle chinesische Medizin (TCM) hat aufgrund ihrer Anwendung durch Emigranten und eine zunehmende Zahl westlicher Anwender ausserhalb Chinas eine nicht zu unterschätzende Bedeutung $[1,2]$. Die völlig eigenständige und aus naturwissenschaftlicher Sicht nicht nachvollziehbare Theorie hat zur Folge, dass die TCM von der «konventionellen» Medizin als unplausibel oder veraltet angesehen und ihre Effektivität in Zweifel gezogen wird [3]. Eine Vielzahl von Fallserien und Studien sowie einzelne randomisierte Studien beschreiben jedoch beträchtliche therapeutische Erfolge, vor allem bei chronischen und funktionellen Erkrankungen [z. B. 4, 5]. 1991 wurde in Kötzting im Bayerischen Wald eine Klinik für traditionelle chinesische Medizin eröffnet, in der ein Team von zehn chinesischen Ärzten der Universität Peking mit - vor allem diagnostischer und zum Teil therapeutischer - Unterstützung von sechs deutschen Ärzten Patienten stationär versorgt. Die sprachliche Kommunikation wird durch zwei Übersetzer sichergestellt. Die Klinik verfügt über 76 Betten. Die gesetzlichen Krankenkassen erstatten die stationäre Behandlung unter der Auflage einer wissenschaftlichen Begleitung. Die Kassenpatienten werden von den behandelnden Ärzten überwiesen.

\section{Methodische Besonderheiten}

Allgemeine Ziele und Methoden der Studie wurden in Teil 1 des Erfahrungsberichts ausführlich beschrieben [6]. Zusätzlich zu der Gesamtauswertung aller Patienten wird für die vorliegende Darstellung eine Auswertung der folgenden Diagnosegruppen vorgestellt:

1. Migräne (ICD-Diagnose 346),

2. Affektionen des peripheren Nervensystems (ICD 350-359),

3. Sonstige Erkrankungen der oberen Luftwege (ICD 470-478) und chronisch obstruktive Lungenkrankheiten (ICD 490-496),

4. Krankheiten des Magens und Duodenums (ICD 531-537), nichtinfektiöse Enteritis und Colitis (ICD 555-558) sowie sonstige Krankheiten des Darms (ICD 564, 569),

5. Arthropathien (ICD 710-719), Dorsopathien (ICD 720-724) und Rheuma, ausgenommen des Rückens (ICD 725-729),

6. Symptome, die Kopf, Hals, Nerven, Muskel- und Skelettsystem betreffen (ICD 781, 784),

7. Erkrankungen der Haut (ICD 680-709),

8. Sonstige Diagnosen.

Die Bildung der Subgruppen erfolgte retrospektiv in Absprache mit den Ärzten unter den Gesichtspunkten der Auswertbarkeit, des Vergleichs mit anderen Häusern des Klinikverbunds und medizinischer Interpretierbarkeit.

\section{Ergebnisse Gesamtgruppe}

\section{Anzahl dokumentierter Patienten}

In der 8monatigen Rekrutierungsphase (12/94-7/95) wurden insgesamt 667 Patienten stationär aufgenommen. Bei den poststationä- ren Befragungen schickten 83,5\% (nach 2 Monaten), 72,3\% (nach 6 Monaten) bzw. 65,8\% (nach 12 Monaten) der Patienten ausgefüllte Bögen zurück.

\section{Informationen zu den Patienten}

$72,3 \%$ der Patienten waren Frauen; das Durchschnittsalter betrug 52,4 \pm 14,4 Jahre. Die Patienten litten im Durchschnitt seit 7 Jahren (Median) an ihren Hauptbeschwerden; bei $66,8 \%$ nahmen diese Beschwerden seit Erkrankungsbeginn an Intensität zu.

Die mittlere Beschwerdeintensität (gemessen mit Hilfe einer visuellen Analogskala von $0 \mathrm{~mm}=$ keine Beschwerden bis $100 \mathrm{~mm}=$ unerträgliche Beschwerden) wurde bei Aufnahme im Mittel mit 71,8 $\pm 21,0 \mathrm{~mm}$ angegeben. Auffällig ist die hohe Erwartungshaltung der Patienten trotz der chronischen Beschwerden: So wurde erwartet, dass die Beschwerden nach Behandlung auf einen Wert von $18,3 \pm 16,7 \mathrm{~mm}$ sinken würden. $74,9 \%$ erwarteten die Verbesserung innerhalb eines Monats.

26,2\% der Patienten litten an Erkrankungen des Skeletts, der Muskeln und des Bindegewebes (ICD 710-739), 26,2\% an Erkrankungen des Nervensystems oder der Sinnesorgane (ICD-9-Nummern 320-389) und 19,6\% an nicht näher klassifizierbaren Symptomen. Häufigste Einzeldiagnosen waren Lumbalgien (12,1\%; klassifiziert als ICD-Diagnose 724 = sonstige Affektionen des Rückens), Migräne (11,4\%; ICD 346) und Schmerzsyndrome im Kopf-/Nackenbereich $(8,8 \%$; ICD $784=$ Symptome, die Kopf und Hals betreffen). Neben der Hauptdiagnose lagen im Mittel 2,61 Nebendiagnosen pro Patient vor. Die mittlere Aufenthaltsdauer betrug 27 Tage.

\section{Therapeutische Leistungen}

Behandlungsschwerpunkte der Klinik sind Akupunkturbehandlungen (bei 99,7\% aller Patienten) und chinesische Arzneimitteltherapie $(98,8 \%) .55,5 \%$ der Patienten erhielten Tuinabehandlungen, 23,2\% bekamen Qi-Gong-Übungen verordnet. Neben diesen Methoden der traditionellen chinesischen Medizin nahmen 28,7\% der Patienten an Ernährungsvorträgen teil, 40,9\% erhielten Spezialdiäten, 24,8\% Gesprächstherapie, 40,8\% erhielten Neuraltherapie und 23,6\% eine homöopathische Behandlung. Eine ausführliche Auflistung der therapeutischen Leistungen in den einzelnen Subgruppen findet sich in Tabelle 3 .

\section{Unerwünschte Therapiewirkungen}

Unerwünschte Therapiewirkungen traten bei 63 (9,4\%) Patienten auf. Am häufigsten wurden reversible Transaminasenerhöhungen (42 Patienten) beobachtet. Gastrointestinale Symptome wurden in sechs Fällen dokumentiert.

\section{Effektivitätsindikatoren}

Als gut oder sehr gut beurteilten den Therapieerfolg 50,3\% der Patienten bei Entlassung, 49,1\% nach zwei, 50,0\% nach sechs und 55,8\% nach 12 Monaten. Die Ärzte beurteilten in 58,7\% der Fälle die Beschwerden der Patienten bei Entlassung als um mindestens $25 \%$ gebessert. $62,1 \%$ der Nebendiagnosen wurden als gebessert beurteilt. Die Intensität der Hauptbeschwerden sank 
von $71,8 \pm 21,0 \mathrm{~mm}$ bei Aufnahme auf $42,6 \pm 25,5 \mathrm{~mm}$ bei Entlassung. Nach zwei Monaten betrugen die Werte 45,6 $\pm 27,6 \mathrm{~mm}$, nach sechs Monaten 49,6 $\pm 26,8 \mathrm{~mm}$ und nach 12 Monaten $49,6 \pm 26,9 \mathrm{~mm}$.

Bezüglich der Lebensqualität zeigten sich insbesondere in den Dimensionen Körper und Psyche der Münchener Lebensqualität-Dimensionen-Liste (MLDL [7)) und des Fragebogens Alltagsleben [8] weitgehend stabile Effekte der stationären Behandlung. Die emotionale Befindlichkeit (bestimmt mit Hilfe des Profile of Mood States [9]) verbesserte sich zwar im Verlauf des stationären Aufenthaltes deutlich, war poststationär jedoch schnell rückläufig und entsprach nach 12 Monaten dem Ausgangsniveau vor Behandlung. Eine eingehendere Beschreibung von Ergebnissen findet sich bei den Subgruppen.

Etwa ein Drittel der Patienten gab an, dass der Medikamentenverbrauch seit der stationären Behandlung niedriger sei als vorher (Tab. 1). In der Gesamtzahl der Medikamente schlug sich dies jedoch nicht deutlich nieder. Die überwiegende Mehrheit der Patienten führte die jeweils verordneten Therapien sowie die Voll- werternährung poststationär weiter. Im Jahr nach der stationären Behandlung entwickelte etwa ein Drittel der Patienten neue Beschwerden, über die jedoch keine weiteren Angaben vorliegen. Nach 12 Monaten hatten 41,6\% eine andere Behandlung erhalten, über die Hälfte davon mit gutem Erfolg. Die Zahl der Patienten, die ihrem Arbeitsplatz in den letzten Monaten ferngeblieben waren, war sechs Monate nach Therapie deutlich geringer als bei Aufnahme. Für einen Grossteil der Patienten traf jedoch diese Frage wegen Nichterwerbsfähigkeit nicht zu.

\section{Ergebnisse Subgruppen}

Bei der Analyse der Subgruppen fallen erhebliche Unterschiede bezüglich des Geschlechts, Bestehensdauer der Beschwerden, therapeutischer Leistungen, Therapieerfolge und Rücklaufquoten auf (Tab. 2, 3; Abb. 1-3). So ist unter den Migränepatienten der Frauenanteil mit $86,8 \%$ am höchsten und auch die durchschnittliche Zeitdauer seit Bestehen der Beschwerden mit 22 Jahren mit Ab-

Tab. 1. Inanspruchnahmeverhalten, neue Beschwerden und Arbeitsunfähigkeit

\begin{tabular}{|c|c|c|c|c|}
\hline & Bei Aufnahme & Nach 2 Monaten & Nach 6 Monaten & Nach 12 Monaten \\
\hline $\mathrm{n}$ & 667 & 557 & 482 & 439 \\
\hline \multicolumn{5}{|l|}{ Medikamente } \\
\hline Keines & $42,5 \%$ & $48,1 \%$ & $43,2 \%$ & $41,9 \%$ \\
\hline $1-2$ & $15,7 \%$ & $16,5 \%$ & $22,2 \%$ & $16,8 \%$ \\
\hline $3-5$ & $34,6 \%$ & $30,7 \%$ & $28,2 \%$ & $34,9 \%$ \\
\hline$>6$ & $7,1 \%$ & $4,7 \%$ & $6,4 \%$ & $6,4 \%$ \\
\hline Med.-Verbrauch seit Entl. gesunken & & $33,5 \%$ & $40,1 \%$ & $35,1 \%$ \\
\hline Neue Beschwerden hinzugekommen & & $25,0 \%$ & $30,6 \%$ & $35,1 \%$ \\
\hline Verordnete Therapie weiter & & $92,7 \%$ & $88,0 \%$ & $85,6 \%$ \\
\hline Weiterhin Vollwertkost gegessen & & $86,0 \%$ & $84,4 \%$ & $86,3 \%$ \\
\hline Andere Behandlungen bekommen & & $19,3 \%$ & $37,7 \%$ & $41,6 \%$ \\
\hline Davon mit gutem Erfolg & & davon $54,6 \%$ & davon $52,4 \%$ & davon $52,9 \%$ \\
\hline Dem Arbeitsplatz ferngeblieben & \multicolumn{2}{|l|}{ letzte 3 Monate } & \multicolumn{2}{|l|}{ seit letzter Befr. } \\
\hline $\mathrm{Nie}$ & \multicolumn{2}{|l|}{$22,6 \%$} & \multicolumn{2}{|l|}{$52,4 \%$} \\
\hline$<1$ Woche & \multicolumn{2}{|l|}{$19,5 \%$} & \multicolumn{2}{|l|}{$21,1 \%$} \\
\hline 1 Woche-1 Monat & \multicolumn{2}{|l|}{$34,0 \%$} & \multicolumn{2}{|l|}{$14,5 \%$} \\
\hline$>1$ Monat & \multicolumn{2}{|l|}{$23,9 \%$} & \multicolumn{2}{|l|}{$11,9 \%$} \\
\hline Keine Angabe & \multicolumn{2}{|l|}{334} & \multicolumn{2}{|l|}{255} \\
\hline
\end{tabular}

Tab. 2. Diagnosen, Anzahl, Geschlecht, Beschwerdedauer und Therapieerfolg in den einzelnen Diagnosegruppen

\begin{tabular}{|c|c|c|c|c|c|c|}
\hline \multirow[t]{2}{*}{ Erkrankung } & \multirow[t]{2}{*}{ ICD } & \multirow[t]{2}{*}{$\mathrm{n}(\%)$} & \multirow{2}{*}{$\begin{array}{l}\text { Weiblich } \\
(\%)\end{array}$} & \multirow{2}{*}{$\begin{array}{l}\text { Beschw. seit } \\
\left(\text { Jahre }^{a}\right)\end{array}$} & \multirow{2}{*}{$\frac{\% \text { Therapieerfolg }}{\text { Entlassung }}$} & \multirow{2}{*}{$\begin{array}{l}\text { gut/sehr gut } \\
12 \text { Monate } \\
\left(\mathrm{FU}^{\mathrm{b}}\right)\end{array}$} \\
\hline & & & & & & \\
\hline Migräne & 346 & $76(11,4)$ & 86,8 & $22,0 \pm 12,7$ & 69,1 & $61,8(74)$ \\
\hline Peripheres Nervensystem & $350-359$ & $70(10,5)$ & 68,6 & $9,3 \pm 9,4$ & 44,6 & $56,1(63)$ \\
\hline Obere Luftwege/Lunge & $470-478,490-496$ & $56(8,4)$ & 64,3 & $10,8 \pm 7,8$ & 52,9 & $61,1(66)$ \\
\hline Magen/Darm & $531-537,555-558,564,569$ & $45(6,7)$ & 71,1 & $9,3 \pm 8,5$ & 41,4 & $71,4(62)$ \\
\hline Stütz-/Bew.app. & $710-729$ & $176(26,3)$ & 71,1 & $9,0 \pm 9,2$ & 52,8 & $54,3(69)$ \\
\hline Kopf/Nacken & $781 / 784$ & $91(13,6)$ & 72,5 & $11,0 \pm 11,2$ & 43,7 & $47,6(70)$ \\
\hline Haut & $680-709$ & $21(3,1)$ & 71,4 & $10,2 \pm 10,5$ & 68,4 & $54,6(52)$ \\
\hline Sonstige & & $133(19,9)$ & 68,2 & $8,0 \pm 9,0$ & 42,8 & $50,7(62)$ \\
\hline
\end{tabular}

${ }^{\text {a }}$ Mittelwert \pm Standardabweichung.

${ }^{\mathrm{b}} \mathrm{FU}=$ Rücklaufquote (Patienten mit zurückgeschickten Fragebogen/alle Patienten in \%). 
stand am längsten, während bei allen anderen Gruppen die Werte mit 9 bis 11 Jahren Krankheitsdauer relativ ähnlich sind.

Erwartungsgemäss finden sich indikationabhängig erhebliche Unterschiede in der Anwendungshäufigkeit eines Teils des therapeutischen Spektrums (Tab. 3). Während Akupunktur und chinesische Arzneimitteltherapie bei fast allen Patienten angewendet werden, erfolgt die Behandlung mit Tuinamassagen bei Erkrankungen des Stütz- und Bewegungsapparates besonders häufig $(75,3 \%)$. Bei Hauterkrankungen wird sie dagegen nur bei wenigen Patienten (9,5\%) eingesetzt. Qi-Gong-Übungen wurden besonders bei Patienten mit Affektionen des peripheren Nervensystems eingesetzt (42,9\%).

Spezialdiäten wurden am häufgsten bei Magen-Darm-Erkrankungen $(68,9 \%)$ durchgeführt, am seltensten bei Migräne (20\%), Neuraltherapie am häufigsten bei Affektionen des peripheren Nervensystems $(65,7 \%)$ und am seltensten bei Hauterkrankungen $(23,8 \%)$.
Bei Migräne- und Hautpatienten (cave: geringe Gruppengrösse bei Hauterkrankungen) scheint die Besserung der Beschwerden während des stationären Aufenthaltes besonders ausgeprägt zu sein (siehe Abb. 1); dementsprechend bewerten die Patienten den Therapieerfolg hoch. Sehr auffällig sind einzelne Unterschiede zwischen der Bewertung des Therapieerfolgs bei Entlassung sowie nach 12 Monaten. Während der Anteil der Patienten, die den Therapieerfolg als gut bzw. sehr gut bewerten, bei Migräne und Hauterkrankungen nach 12 Monaten deutlich niedriger als bei Entlassung liegen, ist dies bei Patienten mit Magen-Darm-Erkrankungen umgekehrt: Während bei Entlassung nur 41,4\% die Therapie als erfolgreich ansehen, liegt die Quote nach 12 Monaten bei 71,4\% (siehe Tab. 2, vorletzte und letzte Spalte). Die Gruppe der MagenDarm-Patienten ist ausserdem die einzige, bei denen sich die Intensität der Hauptbeschwerden kontinuierlich verringert.

Tab. 3. Häufigkeit (in \%) der Anwendung einzelner Therapieformen in den verschiedenen Diagnosegruppen. Aufgeführt sind nur Therapien, die in mindestens einer Diagnosegruppe in mehr als 20\% der Patienten zur Anwendung kamen.

\begin{tabular}{|c|c|c|c|c|c|c|c|c|}
\hline \multirow[t]{3}{*}{ Therapie } & \multicolumn{8}{|c|}{ Diagnosegruppe (n) } \\
\hline & 1 & 2 & 3 & 4 & 5 & 6 & 7 & 8 \\
\hline & $\begin{array}{l}\text { Migräne } \\
(75)\end{array}$ & $\begin{array}{l}\text { peripher. NS } \\
(70)\end{array}$ & $\begin{array}{l}\text { ob. Luftwege/ } \\
\text { Lunge } \\
(56)\end{array}$ & $\begin{array}{l}\text { Magen/Darm } \\
(45)\end{array}$ & $\begin{array}{l}\text { Stütz-/Bew.- } \\
\text { Apparat } \\
(174)\end{array}$ & $\begin{array}{l}\text { Kopf/Hals } \\
(91) \\
\end{array}$ & $\begin{array}{l}\text { Haut- } \\
\text { erkrank. } \\
(21)\end{array}$ & Sonstige \\
\hline \multicolumn{9}{|l|}{ TCM-Therapie } \\
\hline Akupunktur & 100,0 & 100,0 & 98,2 & 100,0 & 100,0 & 100,0 & 95,2 & 100,0 \\
\hline Chin. Arzneimittel & 100,0 & 100,0 & 100,0 & 97,8 & 96,6 & 98,9 & 100,0 & 100,0 \\
\hline Tuina-Behandlung & 57,3 & 35,7 & 39,3 & 48,9 & 75,3 & 64,8 & 9,5 & 49,2 \\
\hline Qi Gong & 8,0 & 42,9 & 8,9 & 15,6 & 25,9 & 25,3 & 9,5 & 27,3 \\
\hline \multicolumn{9}{|c|}{ Westliche Komplementärmedizin } \\
\hline Spezialdiäten & 20,0 & 45,7 & 44,6 & 68,9 & 39,1 & 39,6 & 57,1 & 40,2 \\
\hline Neuraltherapie & 24,0 & 65,7 & 37,5 & 26,7 & 43,7 & 54,9 & 23,8 & 31,1 \\
\hline Gesprächstherapie & 21,3 & 25,7 & 17,9 & 33,3 & 19,0 & 29,7 & 28,6 & 30,3 \\
\hline Mikrobiol. Therapie & 8,0 & 10,0 & 19,6 & 31,1 & 8,6 & 11,0 & 23,8 & 10,6 \\
\hline Inhalationen & 9,3 & 7,1 & 42,9 & 2,2 & 12,1 & 4,4 & 9,5 & 11,4 \\
\hline
\end{tabular}

Abb. 1. Intensität der Hauptbeschwerde (Mittelwert und 95\%-Konfidenzintervall; visuelle Analogskala von $0 \mathrm{~mm}=$ keine Beschwerden bis $100 \mathrm{~mm}$ = unerträgliche Beschwerden) bei Aufnahme (Aufn), Entlassung (Entl) und im Follow-up nach 2 (2 Mon), 6 (6 Mon) und 12 Monaten (12 Mon) in den einzelnen Diagnosegruppen (VAS; 0 = keine Beschwerden bis $100 \mathrm{~mm}=$ unerträgliche Beschwerden).

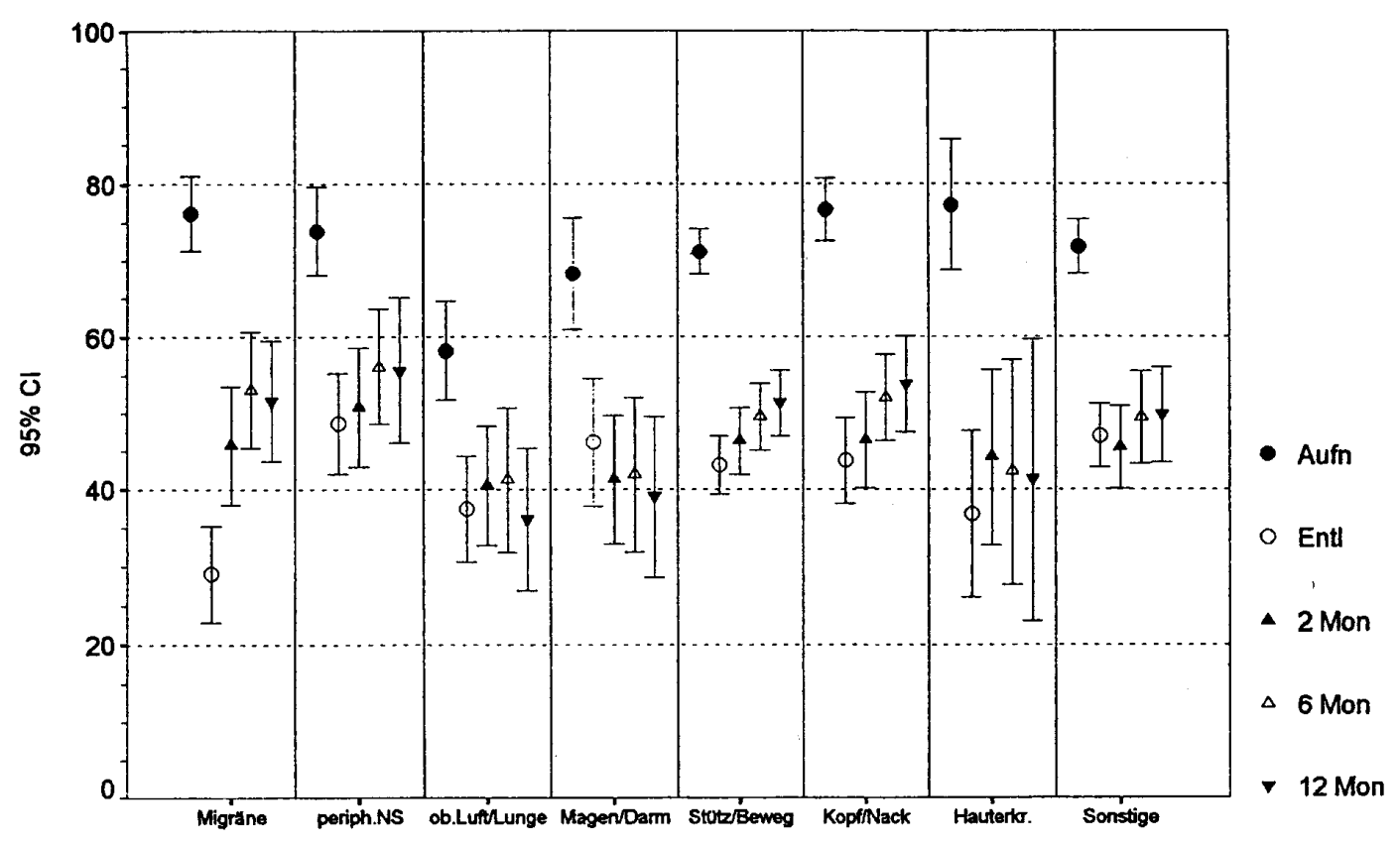


Abb. 2. Lebensqualität $-\mathrm{Zu}$ friedenheit in der Dimension Psyche der MLDL (Mittelwert und 95\%-Konfidenzintervall) bei Aufnahme (Aufn), Entlassung (Entl) und im Follow-up nach 2 (2 Mon), 6 (6 Mon) und 12 Monaten (12 Mon) in den einzelnen Diagnosegruppen.
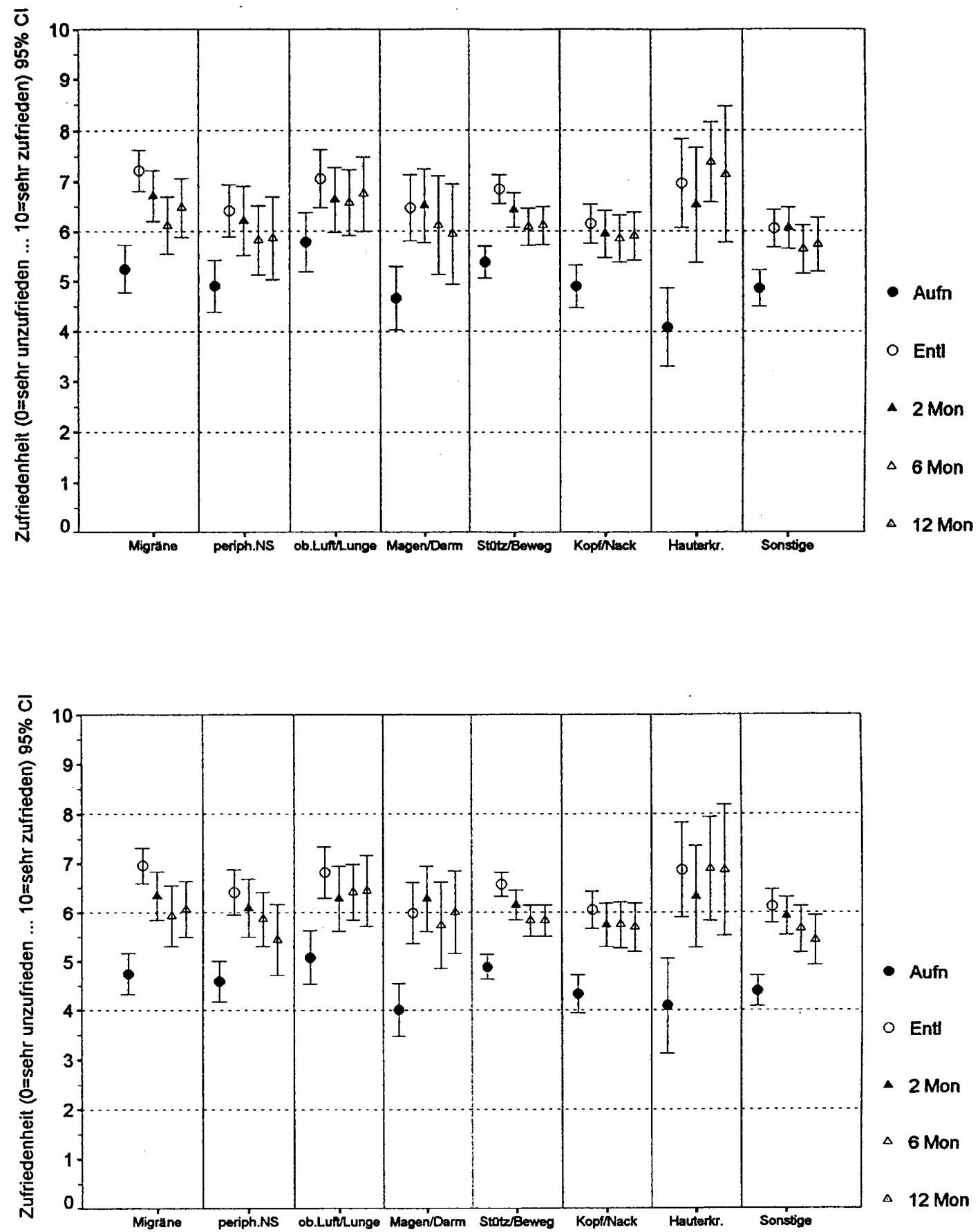

Abb. 3. Lebensqualität - Zufriedenheit in der Dimension Physis der MLDL (Mittelwert und 95\%-Konfidenzintervall) bei Aufnahme (Aufn), Entlassung (Entl) und im Follow-up nach 2 (2 Mon), 6 (6 Mon) und 12 Monaten (12 Mon) in den einzelnen Diagnosegruppen
Die Abbildungen 2 und 3 zeigen die Ergebnisse zur Lebensqualität mit der MLDL in den Dimensionen «Psyche» und «Physis». Ähnlich den Ergebnissen zu Intensität der Hauptbeschwerden kommt es auch in diesen Bereichen zu einer deutlichen und anhaltenden Verbesserung, obwohl die bei der Entlassung besonders günstigen Werte im Follow-up bei der Mehrzahl der Krankheitsgruppen wieder rückläufig sind. Besonders gute und stabile Ergebnisse zeigen sich auch bezüglich der Lebensqualität bei den Hauterkrankungen, während die bei Entlassung sehr guten Behandlungsergebnisse bei Migräne nicht in vollem Masse Bestand haben. Die 12-Monats-Werte sind jedoch etwas günstiger als die Werte nach 6 Monaten.

Beobachtungsstudien im Rahmen eines naturheilkundlichen Klinikverbunds (Teil II)

\section{Diskussion}

Im Gegensatz zu Teil I der Artikelserie, in dem nur einzelne Ergebnisse der ersten Dokumentationsphase der einzelnen Häuser dargestellt und übergreifend verglichen wurden, gibt der vorliegende Artikel einen weitergehenden Einblick in die Resultate, die bei organisatorisch adäquater Durchführung und geeignetem Patientenkollektiv durch eine diagnoseunspezifische prospektive Dokumentation erarbeitet werden können. Während die gemeinsame Auswertung aller Patienten lediglich einen Gesamteindruck der Krankheitsverläufe während und nach Behandlung erlaubt, ermöglicht die Bildung von Diagnosegruppen bereits eine differen-

Forsch Komplementärmed 1998;5:81-86 
ziertere Beurteilung. Dabei wird deutlich, dass die verwendeten Messinstrumente trotz ihrer Einfachheit durchaus sensitiv in der Unterscheidung einzelner Krankheitsgruppen sind. So ergeben sich in den Abbildungen 1 bis 3 bezüglich der globalen Beurteilung der Intensität der Hauptbeschwerde und Lebensqualität zum Teil je nach Krankheitsgruppe vollkommen unterschiedliche, über die verschiedenen Beurteilungsmethoden hinweg jedoch konsistente Verläufe.

Die Rücklaufquoten im Follow-up sind mit 83,5\% (nach 2 Monaten), 72,3\% (nach 6 Monaten) und 65,8\% (nach 12 Monaten) akzeptabel. Die Erfahrungen, die inzwischen über mehrere Jahre gemacht wurden, deuten darauf hin, dass sich höhere Rücklaufquoten im Rahmen der gegebenen Möglichkeiten kaum erreichen lassen. Eine gewisse Positivselektion (also Beantwortung tendenziell eher durch Patienten, die vom Klinikaufenthalt mehr profitiert haben) ist anzunehmen (siehe Teil IV der Artikelserie). Ein weiteres Problem stellt der relativ hohe Anteil «weiterer Behandlungen» mit zunehmender Dauer des Follow-ups dar. Dabei bleibt unklar, ob diese Behandlungen wegen der Hauptbeschwerde oder wegen anderer Erkrankungen erfolgten. Es sollte aber damit gerechnet werden, dass zumindest ein Teil der Patienten wegen Beschwerden behandelt wurde, die für die Beobachtungsstudie relevant waren, und diese Behandlungen die Ergebnisse zumindest bei einem Teil der Parameter beeinflussen.

In der derzeit laufenden Dokumentation wird versucht, die (kom- plementären und konventionellen) Therapien, die die Patienten vor, während und nach dem stationären Aufenthalt erhalten, ausführlicher zu erfassen, da hierzu bisher keine klaren Aussagen gemacht werden können. Ohne den Zugriff auf Daten der Krankenversicherungen lassen sich jedoch zuverlässige Aussagen zu Medikamentenverbrauch, Inanspruchnahme medizinischer Leistungen und Arbeitsunfähigkeit nur schwer ermitteln. Eine enge Zusammenarbeit mit den Hausärzten der Patienten wäre zwar theoretisch ein Lösungsansatz, lässt sich aber praktisch aus vielerlei Gründen (z. B. grosses Einzugsgebiet, Probleme bezüglich Bereitschaft, Daten bereitzustellen, und bezüglich Erhebungsqualität) nicht verwirklichen.

Trotz dieser Einschränkungen belegen die präsentierten Daten auf verschiedenen Ebenen (Beschwerdeintensität, Erfolgsbewertung, Lebensqualität, in eingeschränktem Masse auch Arbeitsunfähigkeit), dass die Patienten - zumindest aus ihrer eigenen Sicht - im Schnitt vom Klinikaufenthalt eindeutig profitieren. Selbstverständlich kann aus den Ergebnissen nicht abgeleitet werden, inwieweit Placeboeffekte oder sonstige Kureffekte, die nicht speziell mit der TCM-Therapie zusammenhängen, für die beobachteten Verbesserungen verantwortlich sind. Ebensowenig kann beurteilt werden, ob die Therapie in der TCM-Klinik gleich, weniger oder mehr wirksam und effizient ist wie andere stationäre oder ambulante Therapieangebote. Dies kann nur in Vergleichsstudien, die auf definierte Patientengruppen beschränkt bleiben, beantwortet werden.

\section{Literatur}

1 Harper J: Traditionel Chinese medicine for eczema. BMJ 1994;308:489-490.

2 Kao FF: The impact of Chinese medicine on America. Am J Chin Med 1992;20:1-16.

3 National Council Against Health Fraud: Acupuncture: The position paper of the National Council against Health Fraud. Clin J Pain 1991;7:162-166

4 Sheehan MP, Rustin MHA, Atherton DJ, Buckley C, Harris DJ, Brostoff J, Ostlere L, Dawson A: Efficacy of traditional Chinese herba therapy in adult atopic dermatitis. Lancet 1992;340:13-17.

5 Vray M, Attali JR: Randomized study of gliben- clamide versus traditional Chinese treatment in type 2 diabetic patients. Chinese-French Scientific Committee for the Study of Diabetes. Diabete Metab 1995;21:433-439.

6 Melchart D, Gaisbauer M, Brenke R, Riker U, Liao JZ, Hager S, Linde K, Weidenhammer W: Beobachtungsstudien im Rahmen eines naturheilkundlichen Klinikverbunds. Teil I: Methoden und Übersicht der Ergebnisse in den beteiligten Kliniken. Forsch Komplementärmed 1998;5:18 25.

7 Heinisch M, Ludwig M, Bullinger M: Psychometrische Testung der Münchener LebensqualitätDimensionen Liste (MLDL); in Bullinger M,
Ludwig M, von Steinbüchel N (Hrsg): Lebensqualität bei kardiovaskulären Erkrankungen. Göttingen, Hogrefe, 1991, pp 73-90.

8 Bullinger M, Kirchberger I, von Steinbüchek N: Der Fragebogen Alltagsleben - ein Verfahren zur Erfassung der gesundheitsbezogenen Lebensqualität. Z Med Psychol 1988;3:121-131.

9 Bullinger M, Heinisch M, Ludwig M, Geier S: Skalen zur Erfassung des Wohlbefindens: Psychometrische Analysen zum «Profile of Mood States» (POMS) und zum «Psychologica General Well-Being Index» (PGWB). Z Differ Diagn Psychol 1990;11:53-61. 